\title{
INMIGRACIÓN, POLÍTICA LINGÜÍSTICA Y SISTEMA EDUCATIVO
}

\author{
FRANCISCO FERNÁNDEZ GARCíA \\ Universidad de Jaén \\ Grupo de Investigación ALTYA (Análisis lingüístico: teoría y aplicaciones) \\ fcofer@ujaen.es
}

\begin{abstract}
Resumen
El presente trabajo somete a análisis las formas en que puede encararse el tratamiento de la situación lingüística de los inmigrantes que se incorporan a nuestro sistema educativo, en busca de su máxima integración social. En relación con ello, analiza algunas de las directrices que se han seguido en otros países con mayor tradición como receptores de inmigrantes e incide en la conveniencia de que dicho tratamiento no se base exclusivamente en la enseñanza de la lengua de acogida sino que tome también en consideración las lenguas de origen.
\end{abstract}

PALABRAS CLAVE: inmigración, política lingüística, sistema educativo, integración

\begin{abstract}
This work analyses the different possible approaches to the linguistic competence of those immigrants that enter our Spanish educational system so that their maximum social integration can be reached. Regarding this issue, this work both studies those strategies that have been adopted in some other countries where most immigrants have settled and insists on the importance of not only taking into account the target language learning, but also the corresponding mother tongue background.
\end{abstract}

KEY WORDS: immigration, linguistic policies, educational system, integration

\section{Introducción}

El fenómeno de la inmigración ha transformado la faz de la sociedad española en muy pocos años. En efecto, una nación que vio emigrar constantemente a sus ciudadanos durante varias décadas se ha convertido, en escasos lustros, en destino de llegada de un importante caudal de inmigrantes de la más variada procedencia. Este proceso, acaecido tras el despegue de la economía española una vez consolidada la transición democrática, ha tenido consecuencias de variada naturaleza en muy distintos niveles de la estructura socioeconómica de la nación, como el mercado laboral, los servicios sanitarios o el sistema educativo. En este último campo, hallamos una escuela que, acostumbrada a un alumnado tradicionalmente homogéneo en cuanto a su origen, de pronto encuentra en sus aulas una realidad diferente a la que debe hacer frente del mejor modo posible. Esa nueva realidad implica a niños de distintos orígenes geográficos y, 
lo que es más importante, culturales y lingüísticos; y ello ocurre no sólo en grandes ciudades sino incluso en pequeñas poblaciones y zonas rurales.

Las administraciones públicas, en general, no pueden mirar hacia otro lado ante esta nueva realidad y, desde luego, no puede hacerlo la escuela pública. Pero, indudablemente, se trata de una cuestión compleja cuyo adecuado tratamiento exige un profundo conocimiento previo de la situación. A dicho conocimiento se orientan iniciativas como el proyecto de investigación en el que se inscribe este artículo, desde el que se está trabajando en el conocimiento profundo y detallado de la situación lingüística existente en la ciudad de Jaén a raíz del aumento de la población inmigrante ${ }^{1}$. La investigación se inscribe en una línea de corte sociolingüístico, específicamente en la vertiente de la sociología del lenguaje, trabajando al mismo tiempo en el plano gramatical desde el prisma de la tipología lingüística y con la lingüística aplicada y la pragmática intercultural como puntos de referencia adicionales. El objetivo global es obtener un completo conocimiento de la situación lingüística existente en el ámbito geográfico mencionado: qué lenguas se hablan, cuáles son sus características, cómo perciben los inmigrantes la situación de contacto lingüístico, etc.

En ese marco, nos planteamos en el presente trabajo una reflexión acerca de cómo puede encarar nuestro sistema educativo (hablamos del caso español, y particularmente el andaluz) la situación ante la que se encuentra, y lo haremos analizando cómo se ha actuado en países que poseen ya una larga tradición en la recepción de inmigrantes. Partiremos, además, de una convicción inicial: las medidas de planificación lingüística que se adopten no deberían ser unidireccionales, dirigidas exclusivamente a la enseñanza del español en un intento de difuminar las diferencias de los colectivos de inmigrantes, sino que deberían basarse en una bidireccionalidad que tome también en consideración las lenguas de origen de nuestros nuevos conciudadanos.

1 Se trata del proyecto "Catálogo de lenguas y censo lingüístico de la población extranjera estable de la ciudad de Jaén”, financiado por la Consejería de Innovación, Ciencia y Empresa de la Junta de Andalucía y los Fondos FEDER de la Unión Europea (ref. P07-HUM-03057). El equipo investigador está formado por Francisco Fernández García (investigador principal), Elena Felíu Arquiola, Carmen Conti Jiménez y Marta Torres Martínez. Asimismo, participan en el proyecto dos técnicos: Adoración Herrera Jerónimo y Juan Antonio Chica Sabariego. En su ejecución se está contando con la colaboración de diversas instituciones públicas y privadas, como el Excmo. Ayuntamiento de Jaén, la Subdelegación del Gobierno en Jaén, el Instituto Andaluz de la Mujer la Asociación Jaén Acoge, la Asociación de Trabajadores Marroquíes en España (ATIME Jaén), la Asociación de Mujeres Inmigrantes (AMIN), el Centro de Educación Permanente Almadén, Cruz Roja Española, el Colectivo de Mauritanos en España, la Fundación Don Bosco, la Asociación Entorno Mujer y la Asociación para la Integración: Diálogo, Participación y Valores (ASIJAÉN). La investigación, por otro lado, forma parte de las actividades del Grupo de Investigación ALTYA (Análisis lingüístico: teoría y aplicaciones), financiado por la Junta de Andalucía (HUM-834) e incentivado por el Proyecto Campus de Excelencia internacional en patrimonio cultural y natural. 


\section{Sistema educativo e integración}

Antes de entrar en materia, hemos de detenernos en un concepto clave que sale inevitablemente al paso cuando se analiza la conveniencia o inconveniencia de adoptar cualquier tipo de medidas en relación con los colectivos de inmigrados: el de la integración de dichos colectivos en la sociedad de acogida. El problema en este sentido es que, de forma paralela a la opción entre uni- o bidireccionalidad antes planteada, el concepto de integración admite demasiados matices, de manera que

puede ir desde la asimilación al multiculturalismo, es decir, desde entender en el primer caso- que el inmigrante debe desdibujar sus diferencias respecto del nuevo entorno en que se integra hasta concebir -en el segundo caso- que dichas diferencias son un valor para la sociedad, que debe ser cuidado y mantenido. (Fernández García, 2009: 61).

Efectivamente, como veremos a lo largo de estas páginas, se trata de una disyuntiva siempre presente $e^{2}$ y en la que lo más habitual hasta ahora viene siendo el camino de la asimilación, que acostumbra a ser percibida por los estamentos públicos como la vía más adecuada para gestionar la situación de los inmigrados, en general, y más en concreto las cuestiones relacionadas con la política lingüística.

Dicha gestión, en general y específicamente en el plano de la política lingüística, encuentra uno de sus puntos clave y más debatidos en el sistema educativo. Pitkänen, Verma y Kalekin-Fishman (2002: 9) ven claro el motivo de tal importancia, a saber, que la educación se configura con frecuencia como un elemento clave en el proceso de integración. Ahora bien, si existe consenso sobre la necesidad de llevar a cabo ese proceso de integración de los niños y jóvenes procedentes de familias inmigrantes, lo que no está tan claro es el modo mediante el que ha de llevarse a término, en esa disyuntiva entre asimilación y multiculturalismo. En otro lugar hemos expuesto las dos opciones que, a juicio de Moreno Fernández (1998: 222), se plantean en este sentido:

a) el diseño de programas especiales de inmersión, como modelo transitorio, para facilitar el aprendizaje de la nueva lengua; y b) el diseño de programas que incorporen la L1 en el currículo educativo, en busca de la armonía de la cultura de origen y la de destino. (Fernández García, 2009: 69).

No obstante, Moreno Fernández explica que ambas opciones plantean el mismo problema, la necesidad de una alta financiación, circunstancia que lleva

2 Una interesante discusión sobre los modelos ideológicos que subyacen a los diferentes caminos posibles en este sentido puede hallarse en Alcalá Recuerda (2008: 93-97). 
muchas veces a que el sistema educativo acabe mirando hacia otro lado y abandone a su suerte a esos alumnos. En este sentido se expresan, por ejemplo, Barni y Bagna (2008: 294, 305), quienes afirman que en Italia, en ausencia de una legislación específica que proteja las lenguas inmigrantes (como, en general, ocurre en la Unión Europea), la tendencia habitual en el sistema educativo parece ser la de hacer caso omiso de la variedad lingüístico-cultural existente en el alumnado.

\section{El tratamiento de las lenguas de origen}

Son muchos los argumentos que justifican, desde luego, la conveniencia de que los esfuerzos en política lingüística relativos a la inmigración no se centren exclusivamente en la imposición de la lengua de acogida, empezando por motivos carácter humanitario y concluyendo por razones de cohesión social. Pero lo cierto es que resulta innegable la existencia de dificultades objetivas a la hora de abordar el tratamiento de las lenguas de origen y su posible enseñanza en la escuela. El primero y más evidente es su variedad, es decir, el alto número de lenguas que llegan a reunirse incluso en poblaciones de reducido tamaño, como ocurre, por ejemplo, en la ciudad de Jaén, con una población total que, según datos del padrón municipal de abril de 2011, apenas supera los 117.000 habitantes. Pensemos que, cuando se ha tomado contacto (en el trabajo de campo del proyecto de investigación antes mencionado) con un $79 \%$ de la población extranjera adulta de la ciudad de origen no hispanohablante, el número de lenguas encontradas asciende ya a $92^{3}$. No hace falta decir que la situación se complica aún más en lugares con una especial concentración de población inmigrante, pues hemos de tener en cuenta que ese porcentaje de población encuestada en Jaén corresponde a poco más de mil individuos.

A esta dificultad inicial hay que añadir, como señala Edwards (2008: 256), el gran problema de la falsa homogeneidad con que las lenguas de origen de los inmigrantes son vistas en los países de destino, en los que, por ejemplo, se hace referencia genérica a los hablantes de árabe o de chino, sin tomar en consideración la presencia de numerosos y muy diferenciados dialectos (muchas veces ininteligibles entre sí). En este sentido, es reveladora la postura adoptada en el trabajo de Nygren-Junkin (2008: 277-278): tras hacer referencia al chino como novena lengua extranjera más hablada por la población escolar de Gotenburgo, en Suecia, aclara que utiliza chino como "an umbrella term" para denominar a un conjunto de diferentes lenguas (los llamados dialectos chinos).

3 La relación de lenguas halladas fue presentada en Fernández García (2011). 
Así pues, las dificultades objetivas existentes, unidas a la falta de voluntad política en este sentido, hacen que sean infrecuentes las acciones de política lingüística a favor de estas lenguas en los sistemas educativos. Revelador es el caso de Estados Unidos, donde, según explican Tannenbaum y Berkovich (2005: 288), el aprendizaje de las lenguas minoritarias por parte de los niños y jóvenes inmigrantes, más allá de no ser promovido, es manifiestamente coartado, en la medida en que se espera de las segundas y terceras generaciones de inmigrantes que muestren su integración mediante el uso exclusivo del inglés. Este planteamiento, que se repite en muchos lugares del mundo (pongamos, como segundo ejemplo, a los hijos del voluminoso colectivo de rusohablantes de Israel, en cuyos hogares se suele hablar exclusivamente ruso), conduce a situaciones en las que estas segundas generaciones de inmigrantes quedan sumidas en un semianalfabetismo en su lengua materna, con duras repercusiones socioemocionales en muchos casos.

Todo ello es consecuencia de la frecuente percepción, por parte de gobernantes y ciudadanos, de la enseñanza de las lenguas de las minorías inmigrantes como un obstáculo para la integración. Y no deja de llamar la atención que esto ocurra, por ejemplo, en la Unión Europea, donde tanto hincapié se hace en la importancia de defender la variedad lingüística interna. De hecho, como señalan Extra y Gorter (2008: 9, 35), aunque la situación de las lenguas regionales y de las lenguas inmigrantes tiene no pocos aspectos en común, lo cierto es que el tratamiento que a uno y otro grupo se otorga en Europa es, en general, manifiestamente distinto y que el debate sobre la conveniencia -e incluso legitimidad- de la presencia de las lenguas originarias de los colectivos inmigrados en el sistema educativo es arduo, en la medida en que se tiende a considerarlas más una fuente de problemas que una fuente de enriquecimiento.

No obstante, parece claro que, como escribe García Marcos (2008: 152), se impone la necesidad de "programar la coexistencia entre las lenguas" reunidas en una determinada comunidad por el devenir histórico. De este modo, poco a poco van dándose casos, tanto fuera como dentro de Europa, que parecen anunciar un inevitable cambio futuro de la tendencia en la política lingüística. Y dichos cambios acostumbran a aparecer, como es lógico, en países con gran tradición y volumen de comunidades extranjeras (aunque no siempre sea el caso, claro está, como en el ejemplo de Estados Unidos). Muy citada es la situación de Australia. Smolicz y Secombe (2003: 7-10) explican que, tras varias décadas con un sistema educativo férreamente orientado a la asimilación, a partir de los años 80 fue abriéndose un panorama diferente, en el que, con el inglés como lengua general, dejaba de excluirse de la enseñanza al resto de las lenguas allí habladas, situación que se ha ido potenciando posterior- 
mente, tanto en lo tocante a las lenguas aborígenes como a las de los grupos inmigrados. También puede mencionarse el caso del Reino Unido, donde, según Edwards (2008: 261-263), va dándose cabida progresiva en el sistema educativo a algunas de las lenguas más representativas de las comunidades extranjeras, como el árabe o el chino.

Pero el desarrollo de una postura realmente avanzada en este sentido lo muestra el caso de Suecia, país pionero en no pocos aspectos. Nygren-Junkin (2008: 280-288) explica que, desde los años 70, la legislación de ese país contempla la obligación de los colegios de remitir información a los servicios centrales de estadística acerca de sus alumnos inmigrantes, y ello con vistas a un doble objetivo: planificar la enseñanza del sueco como L2 y planificar la enseñanza de las lenguas de origen. En cuanto al segundo aspecto, la enseñanza de la lengua de origen del alumno se contempla como un derecho universal, siempre que el individuo pertenezca a la primera, segunda o tercera generación de inmigrantes y posea ya ciertos conocimientos de la lengua en cuestión (muestra de que es utilizada en el ámbito familiar); por lo demás, la única restricción existente para que se oferte la formación en una lengua determinada es que sea demandada por, al menos, cinco alumnos en el ámbito de un determinado municipio.

El caso sueco (que constituye, sin duda, una muestra paradigmática de respeto a la variedad lingüística, lo que en último término implica respeto a la variedad étnica y cultural) nos conduce a una cuestión de interés: cuando se dan los requisitos mencionados, las familias pueden solicitar la enseñanza en su lengua nativa. Ello traslada la cuestión al ámbito de la política lingüística familiar -utilizando una idea de Spolsky (2004: 42)-, que puede sufrir pocas presiones tan fuertes como las provocadas por una situación de inmigración. En dichas situaciones, los hijos de los inmigrantes, que acostumbran a alcanzar una socialización mayor que la de sus padres, tienden a alejarse de la herencia lingüística; en cualquier caso, no obstante, la decisión clave suele estar en manos de los padres, a saber, la de potenciar o no el mantenimiento de dicha herencia. En este sentido, nuestra investigación sobre la situación en la ciudad de Jaén muestra una manifiesta tendencia: ante la pregunta sobre si estarían dispuestos a que sus hijos aprendieran exclusivamente el español, más del 97\% de los encuestados han respondido que no, argumentando, principalmente, la conveniencia de que puedan mantener vivos los vínculos con la familia residente en su lugar de origen y de que no pierdan el contacto con su cultura de origen (hablan de religión, de costumbres, etc.), así como, en menor grado, las ventajas que en un futuro podría suponerles hablar otra lengua. Los datos son, pues, elocuentes. La población inmigrante siente que su lengua materna 
es parte de su identidad y que, precisamente por ello, las carencias en sus hijos en ese sentido pueden provocar una quiebra en dicha identidad, con las peligrosas consecuencias socioemocionales a las que nos referíamos más arriba. Se trata de un hecho que no debería ser pasado por alto en una situación como la española, en la que, en general, el sistema educativo vuelve la espalda a las lenguas de origen de la población inmigrante.

No deben dejar de señalarse, no obstante, los movimientos que algunos estamentos públicos vienen realizando en esta dirección. En el caso concreto de Andalucía, la Consejería de Educación y Ciencia, en su Plan Andaluz de Educación de Inmigrantes en la Comunidad Autónoma Andaluza (2001), hablaba ya en su objetivo cuarto de facilitar en el sistema educativo el aprendizaje de la lengua materna por parte de la población inmigrante con el objeto de que tales alumnos no se desligaran de sus raíces lingüísticas; posteriormente, en el Plan de Fomento del Plurilingüismo. Una política lingüística para la sociedad andaluza (2005), se vuelve sobre la misma cuestión en varias acciones generales. Lamentablemente, sin embargo, estas iniciativas se quedan, en la mayoría de los casos, en el nivel de las buenas intenciones, sin llegar a plasmarse en acciones decididas.

\section{El tratamiento de la lengua de destino}

Planteábamos al comienzo de este trabajo la doble vertiente existente en el concepto de integración, asimilación frente a multiculturalismo. En el ámbito de la política lingüística, está claro que todos los esfuerzos que se hagan en relación con la introducción de las lenguas minoritarias en el currículo educativo vendrán a cubrir el flanco del multiculturalismo, pero nos queda ahora asomarnos al plano de la asimilación, en este caso plasmada en la enseñanza de la lengua de destino. En este sentido, como afirma García Marcos (2008: 154-155), hay que pensar que el dominio de la lengua de destino para los inmigrados no ha de entenderse como una imposición sino simplemente como la adquisición de la herramienta que permita la interacción social que necesita todo individuo. Por consiguiente, la adecuada planificación de la enseñanza de la lengua mayoritaria como L2 resultará capital para el éxito del conjunto del proceso de integración social del inmigrante.

En el caso de Francia, desde mediados de los noventa se viene desarrollando un programa de enseñanza del francés como L2 (Spolsky, 2004: 70-71); en Australia, por poner un segundo ejemplo, hace décadas que se desarrolla la enseñanza del inglés como L2 en el sistema educativo (Smolicz y Secombe, 2003: 12). Pensemos que es enormemente frecuente el caso de hijos de inmigrantes en cuyo hogar se habla exclusivamente la lengua materna familiar; y 
que esos niños y jóvenes, cuando llegan a una escuela en la que se habla una lengua que no dominan, estarán abocados al fracaso escolar si son abandonados a su suerte. Se trata, pues, de una cuestión de suma importancia para el aparato educativo y para la sociedad en su conjunto, y parece ser que el aumento de la conciencia en este sentido está conduciendo en los últimos años, en el ámbito de la Unión Europea (Extra y Gorter, 2008: 35), a un sustancial aumento del esfuerzo y los recursos destinados a la enseñanza de la lengua mayoritaria como L2. Así, Barni y Bagna (2008: 306-308) explican, respecto del caso italiano, que las acciones lingüísticas que se están adoptando en relación con la inmigración ponen el foco de manera casi exclusiva en la potenciación de la enseñanza del italiano como L2.

No se trata siempre, sin embargo, de un trabajo bien dirigido y planificado, y los sistemas educativos de no pocos países muestran lagunas en este sentido. Así lo denuncian, por ejemplo, Hernández García y Villalba Martínez (2008) respecto del caso español, criticando la inexistencia de un currículo oficial para la enseñanza de la lengua española como L2 en el contexto educativo. Zapico Alonso (2006) incide en la misma carencia, señalando que el inmigrante escolarizado supone un caso muy distinto al de la persona que necesita un español general "de supervivencia": es un estudiante al que le urge integrarse en un sistema educativo y ser capaz de superar una serie de materias académicas específicas. García Marcos (2008: 155-158), por su parte, analizando el caso concreto andaluz, critica que se esté trabajando al margen de una adecuada planificación de la mano de los avances en lingüística aplicada y sociolingüística, de manera que, no pocas veces, se desperdicien esfuerzos dando palos de ciego. Concretamente, realiza estos comentarios en referencia a la actividad de las ATAL (Aulas Temporales de Adaptación Lingüística), experiencia surgida en Almería en 1999 y exportada después al resto de Andalucía y a otras comunidades autónomas, cuyo esfuerzo elogia, pero a las que reconoce más las buenas intenciones que el acierto ${ }^{4}$. También Ortiz Cobo (2007: 1) se muestra crítica con el funcionamiento de las ATAL, denunciando una ausencia de regulación global de su funcionamiento, con una mal entendida flexibilidad que acaba derivando en arbitrariedad.

El mismo García Marcos (2008: 159) realiza algunas propuestas sobre cómo debe orientarse la enseñanza del español como L2 dentro del sistema educativo, incidiendo en la conveniencia de organizar programas intensivos

4 Tras su inicio como experiencia piloto en Almería, la creación de las ATAL a nivel autonómico se contempla en el Plan Andaluz de Educación de Inmigrantes en la Comunidad Autónoma Andaluza (2001). También se alude a ellas, si bien es cierto que sólo de pasada, en el Plan de Fomento del Plurilingüismo. Una política lingüística para la sociedad andaluza (2005). 
antes que prolongados en el tiempo. Y es que las ATAL andaluzas no poseen, desde luego, dicho carácter intensivo. Fernández-Gaytán et alii (2006: 3-7) explican, a propósito del caso de la provincia de Granada, la configuración de estas aulas a base de profesorado itinerante, que va desplazándose de centro en centro para dedicar tan sólo tres horas semanales a cada grupo; y demandan que los profesores puedan dedicar más horas a cada grupo, trabajando en menos centros simultáneamente. En esta línea, Ortiz Cobo (2007: 4) va un paso más allá al proponer abiertamente que los profesores de las ATAL dejen de ser itinerantes para integrarse en los centros.

Un modelo que se sitúa más en esa línea estable e intensiva sugerida es el de las Aulas de Enlace que funcionan desde hace unos años en la enseñanza pública y concertada de la Comunidad de Madrid. Estas aulas suponen un tipo de segregación orientado a acortar lo máximo posible el periodo de integración en el sistema educativo ordinario. Núñez Gómez y Cerrillo Martín (2004) explican que el alumno, en principio, trabaja a tiempo completo en el aula específica y, conforme van aumentando sus destrezas comunicativas, se va procediendo a una integración progresiva (y selectiva en las materias académicas) en el llamado "grupo de referencia", o sea, el grupo ordinario que le corresponde, con la limitación de que el conjunto del proceso no supere los seis meses.

Por otro lado, más allá de recomendar el carácter intensivo de los programas, García Marcos (2008: 159) destaca la importancia de no mezclar indiscriminadamente a los alumnos sino distribuirlos en grupos homogéneos según la tipología lingüística y la red sociolingüística de procedencia. Dicha agrupación podría considerarse contraproducente para la integración social, pero no tiene por qué serlo si pensamos en cursos intensivos, breves en el tiempo; además, hay que considerar que lo urgente es que el alumno disponga de la herramienta comunicativa de la mejor y más rápida manera posible, evitando problemas de integración como, claramente, es el repetido fracaso escolar que se da en estos casos. Lo cierto, desde luego, es que son frecuentes los testimonios que sostienen que uno de los principales obstáculos con que se encuentran estos grupos de trabajo es la heterogeneidad lingüística y cultural de los alumnos (Núñez Gómez y Cerrilo Martín, 2004; Fernández-Gaytán et alii, 2006).

Ahora bien, la ejecución de una propuesta como ésta última implicaría un profundo conocimiento de la situación, del que, en general, los gestores educativos españoles no están en posesión. Precisamente cubrir esa carencia (aunque sólo sea en un ámbito geográfico localizado, el de la ciudad de Jaén) es lo que pretende el proyecto investigador del que surge este trabajo, con su doble línea de indagación: la genético-tipológica, con la elaboración el catálogo de lenguas, y la sociolingüística, con la elaboración del censo de hablantes. 


\section{Conclusiones}

Nos planteábamos al comienzo de este trabajo un análisis del modo en que los sistemas educativos (en general y en concreto el español y el andaluz) pueden gestionar satisfactoriamente las situaciones de multiculturalidad provocadas por los flujos migratorios, que tan apreciablemente se han intensificado en nuestro país en las últimas décadas. Ha quedado de manifiesto, desde luego, que la situación es tan compleja como merecedora de atención, pues el modo en que la escuela aborde la cuestión lingüística tendrá, a buen seguro, relevantes consecuencias sobre el proceso de integración de la población inmigrante. Dicha gestión debería aspirar a articular adecuadamente lo que, en relación con los procesos de integración, hemos llamado tendencias asimiladoras y multiculturalistas, objetivo que, en la práctica, supondría crear los cauces para lograr que se mantenga la vitalidad lingüístico-cultural de origen de la población inmigrante al tiempo que se potencie y acelere suficientemente su competencia en la lengua de destino. Las dificultades, según hemos visto, son significativas en ambos planos pero no, desde luego, insalvables; y constituyen, sin duda, de uno de los grandes desafíos que nuestro tiempo plantea al sistema educativo.

\section{Referencias bibliográficas}

Alcalá Recuerda, E. (2008): "Multilingüismo en las Aulas: procedimientos de inclusión y exclusión”. En Ríos Rojas, A., y G. Ruiz Fajardo (eds.): Didáctica del Español como $2^{a}$ Lengua para Inmigrantes. Sevilla, Universidad Internacional de Andalucía, págs. 92-108.

Barni, M., y C. Bagna (2008): "Immigrant languages in Italy”. En Extra, G., y D. Gorter (eds.), págs. 293-313.

Consejería de Educación y Ciencia de la Junta de Andalucía (2001): Plan Andaluz de Educación de Inmigrantes en la Comunidad Autónoma Andaluza.

Consejería de Educación y Ciencia de la Junta de Andalucía (2005): Plan de Fomento del Plurilingüismo. Una política lingüística para la sociedad Andaluza.

Edwards, V. (2008): "New minority languages in the United Kingdom". En Extra, G., y D. Gorter (eds.), págs. 253-270.

Extra, G. y D. Gorter (2008): "The constellation of languages in Europe: an inclusive approach”. En Extra, G., y D. Gorter (eds.), págs. 3-60.

Extra, G., y D. Gorter (eds.) (2008): Multilingual Europe: Facts and Policies. Berlín, Mouton de Gruyter.

Fernández García, F. (2009): "Bases para la gestión de una situación de multilingüismo derivada de la inmigración", Cultura, lenguaje y representación / Culture, Language and Representation, 7 , págs. 59-76.

Fernández García, F. (2011): "Cuando Babel se encuentra a la vuelta de la esquina: ¿Casi cien lenguas en la ciudad de Jaén?". Comunicación presentada en el III Congreso internacional sobre lenguas y culturas del mundo. Granada, mayo-junio de 2011. 
Fernández-Gaytán, L. et alii (2006): "ATAL: Educar en la diversidad”, Práctica docente, 3, págs. 1-7.

García Marcos, F. J. (2008): Bases de planificación lingüística para Andalucía. Almería, Universidad de Almería.

Hernández García, M. T., y F. Villalba Martínez (2008): "Inmigrants in Spain: sociolinguistic issues", International Journal of the Sociology of Language, 193-194, págs. 177-190.

Moreno Fernández, F. (1998): Principios de sociolingüistica y sociología del lenguaje. Barcelona, Ariel.

Núñez Gómez, E., y M. R. Cerrillo Martín (2004): "Aulas de Enlace: una iniciativa pedagógica, integradora e intercultural”, Tendencias Pedagógicas, 9, págs. 203-215.

Nygren-Junkin, L. (2008): "Immigrant minority languages in Sweden". En Extra, G., y D. Gorter (eds.), págs. 271-292.

Ortiz Cobo, M. (2007): "Segunda Lengua como medio de integración escolar", Revista Iberoamericana de Educación, 41, págs. 1-5.

Pitkänen, P., G. K. Verma y D. Kalekin-Fishman (2002): "Introduction". En P. Pitkänen, D. Kalekin-Fishman y G. K. Verma (eds.): Education and Immigration. Settlement policies and current challenges. Nueva York, Routledge Falmer, págs. 1-10.

Smolicz, J. J., y M. J. Secombe (2003): “Assimilation or pluralism? Changing policies for minority languages education in Australia", Language Policy, 2, págs. 3-25.

Spolsky, B. (2004): Language Policy. Cambridge, Cambridge University Press.

Tannenbaum, M., y M. Berkovich (2005): "Family relations and language maintenance: implications for language educational policies”, Language Policy, 4, págs. 287-309.

Zapico Alonso, L. (2006): “De Aula en Aula: tareas de ESO para la enseñanza del español como lengua vehicular a alumnos inmigrantes", RedELE, 4 